\title{
The investigation of environmental specifics of an autistic child's family system
}

\author{
Tatyana Ozerova ${ }^{1 *}$, Elena Vorobyova $^{2}$ \\ ${ }^{1}$ Southern Federal University, Academy of psychology and pedagogy, 344006 Rostov-on- \\ Don, Russia \\ 2 Don state technical university, Department of Clinical Psychology and Psychophysiology, \\ 344000 Rostov-on-Don, Russia.
}

\begin{abstract}
The paper is devoted to the actual nowadays problem of environmental specifics of an autistic child's family system. The relevance of the chosen theme is grounded in the paper. Particular attention is paid to the analysis of of related to the problem fundamental theoretical concepts of Russian and translated authors. The paper also presents the outcomes of empirical research and displays statistically significant distinction between psychological indicators of the environment of autistic children's families and those of families with ordinary children according to such parameters as: roles, rules, control, cooperation, discipline, acceptance/rejection, emotional bond. Considerable distinction between the type of family cohesion and the type of family adaptation of an autistic child's family system in comparison to the family system of an ordinary child is described. Statistical reliability of correlation between an autistic child's and an ordinary child's family system and characteristics of the type of the family (dysfunctional, semifunctional, functional) is analyzed. The paper provides the analysis of distinction between the level of marriage satisfaction in autistic child's families and in ordinary child's families.
\end{abstract}

As far back as in the middle of the last century autism was considered to be a rare disorder. Nowadays the number of people suffering from it is increasing. According to the World Health Organization in 2016 every $68^{\text {th }}$ child was born with an autism spectrum disorder and it's important to note that the number is still growing by $13 \%$ a year. According to the World Health Organization experts, autistic disorder is a worldwide phenomenon and this is evident from the fact that people on all continents, in every country, suffer from it regardless of their sex, socio-economic status and race. One of the most pressing concerns

\footnotetext{
*Corresponding author: ozta@mail.ru
} 
in child psychiatry now is the problem of childhood autism. It can be explained by a number of reasons:

- first, by certain difficulties in timely diagnosis

- second, by the frequency of the development of the disorder

- third, by the fact that the causes of the disorder are not fully identified.

Modern psychiatry nowadays puts forward the idea of an impact of certain factors on forming an autism spectrum disorder. The supporters of the theory of psychogenesis who share the idea that the origin of autism is the impact of adverse psychological factors, have expressed their view ever since the time of Kanner's discovery of autism.

Today the scientific community hasn't arrived at a common view on the issue, which is another proof of the relevance of the study. Bruno Bettelheim's monograph "The Empty Fortress: Infantile Autism and the Birth of the Self" has won broad acceptance among specialists and the author is considered to be one of the most faithful adherents of the theory of psychogenic origin of autism [2].

The works of canonical classics of family therapy S.Minuchin, M. Bowen, N. Ackerman, C.Whitaker, I. Boszormenyi-Nagy, the Palo Alto Group and others facilitated the emergence of a new paradigm of symptomatic behavior and under the influence of Bertalanffy's general systems theory instead of focusing on each individual family member and further summing up the information to get an overall picture, an idea appeared, that understanding the process of functioning of all the family members as a whole provides an opportunity to understand its individual member. The process during which the cofunctioning of all family members takes place is called the family system [1]. In the life of an individual person, as well as of the society as a whole, the family plays a large and significant role. In the development of each person, the family not only provides personal development, but also plays a leading role in mental disorders and pathogenic situations as it is an important environmental factor for personality development [5]. In the works of Russian and translated family systems psychotherapists, autism is described as a family system symptom along with other symptoms, such as: separation anxiety in a child when entering school, reduced learning capacity at school age, home theft, child's aggressive behavior, marital conflicts, unmotivated anxiety, depression, sexual dysfunction, anorexia, schizophrenia, bronchial asthma, etc.

In the systems approach, the family is treated as a social system that unites a group of people and their relationships, that is, it is a complex of elements and their characteristics, related to each other and having dynamic correlations between them. The family system is prior to its components [3]. The life of a family system is subject to two laws: the law of homeostasis and the law of the development. In case a law is broken, a symptom appears in the system. The carrier of the symptom in the family can be both a child and an adult. A symptom in the family system fulfills certain functions:

a) the morphostatic function is the preservation of the family system in its current state, that is, the preservation of family homeostasis,

b) the morphogenetic function of the symptom is the transformation of the family system (the law of the development).

Russian family psychotherapists describe the parameters and structural elements of the family system: the numerical and personal composition of the family, rules, roles, subsystems, boundaries, myths and legends, interaction stereotypes, stabilizers of the family system, family histories. Breaching one of them can become a reason for appearance of a symptom in one or in several family members [4].

On the basis of the conducted examination and summing up the principal points of family systems theories of Russian and translated authors from various approaches and scholarly traditions, we can conclude that dysfunctions of the following family system parameters constitute the specific features of a family system as an environmental factor for 
autistic children: rigid or blurred boundaries of family subsystems; rigid, pathologizing roles; role conflicts, intergenerational coalitions; family's difficulties while passing a lifecycle stage, or difficulty in moving from one stage of the life-cycle to another; having a family extrajection; intergenerational transmission of interaction patterns, repressed and inherited emotions; signs of the "anniversary syndrome"; family myths; family legends; dysfunctional stabilizers; lack of clear rules and regulations.

But also dysfunctional: emotional bonds, peculiarities of children upbringing, parentchild relationships, marital relations, family adaptation, low level of satisfaction with family life.

In view of the above, in today's society, the study of the characteristics of the family system of autistic children is of particular importance and relevance, and that is the focus of our study, in which 60 families got involved, 30 of which are families with autistic children living in the city of Azov, Rostov region and visiting the Center for psychological, pedagogical, medical and social assistance "Countenance". As well as 30 families with healthy children living in the city of Novocherkassk, Rostov Region, visiting the Development and Leisure Center.

As a hypothesis, we assumed the following:

- in families with autistic children such environmental features as a high level of parental control and a reduced level of emotional acceptance and cooperation may be representative characteristics

- in families with autistic children, a dysfunctional type of family can take place, which is characterized by incoherence of family members and chaotic nature of adaptation.

- the level of satisfaction with marriage in families with autistic children may differ from the level of satisfaction with marriage in families with an ordinary child.

In the present study the following psychodiagnostic techniques were used: "FACES-3 Family Adaptation and Cohesion Scale"; marriage satisfaction test by V.V. Stolin, T.L. Romanova, G.P. Butenko; parental attitude questionnaire by A.Y. Vargi, V.V. Stolin. Mathematical data processing included the use of the following methods: compilation of contingency tables; nonparametric criteria (Mann-Whitney U-test and Kruskal-Wallace criterion); multivariate analysis of variance (DA). For data processing, traditional software programs for statistical data analysis were used: "STATISTICA 10 RU" and "IBM SPSS Statistics 21".

The study revealed the following data:

The environmental characteristics of an autistic children's family system have statistically significant differences $(p<0.05)$ from the environmental characteristics of families with an ordinary child in such parameters as: roles, rules, control, cooperation, discipline, acceptance / rejection, emotional bond.

The data obtained are presented in the comparative diagram in Figure 1

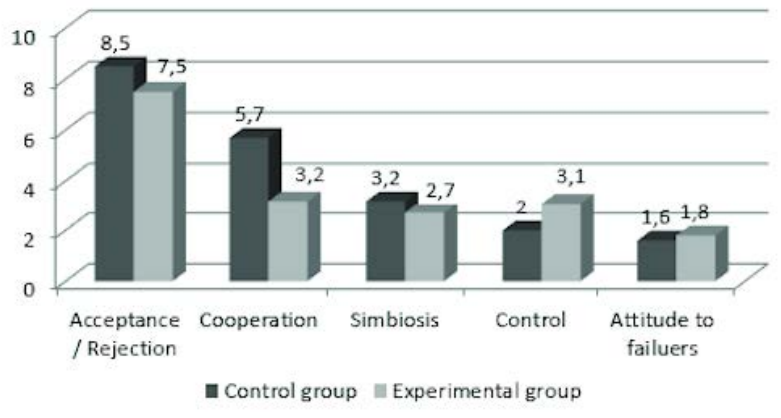

Fig. 1. Statistically significant differences in the environmental characteristics of the family system. Comparative diagram, in points 
Statistically significant differences became apparent through the use of the "Parental Relationship Questionnaire" technique (A.Y. Varga, V.V. Stolin). As seen from the comparative diagram, the statistical significance of the differences was verified by the nonparametric U Mann-Whitney test. As illustrated in the comparative diagram, in the control group there are considerably more families with a high level of "Acceptance / Rejection" and "Cooperation". The experimental group is characterized by an increased level of control and a decreased level of acceptance and cooperation. It is important to note that with increased control, an adult behaves too authoritatively towards the child, imposing strict disciplinary restrictions, demanding unconditional obedience, almost entirely imposing his will on the child. The low level of acceptance and cooperation is a sign that an adult takes little interest in what the child is interested in, does not encourage the child's independence and initiative, does not assess the ability of the child.

Using "The Family Adaptation and Cohesion Questionnaire (FACES-3)" (D.K. Olson, J. Portner, I. Lavi) technique, we studied the levels of cohesion and adaptation of the family system, which revealed the presence of statistically significant differences in the distribution in the control and experimental groups. In the autistic children's family system, a disengaged type of family cohesion statistically $(\mathrm{p}<0.05)$ prevails, thus distinguishing this system from an ordinary child's family system, in which the connected type of family system predomonates. The results are shown by the diagram in Figure2

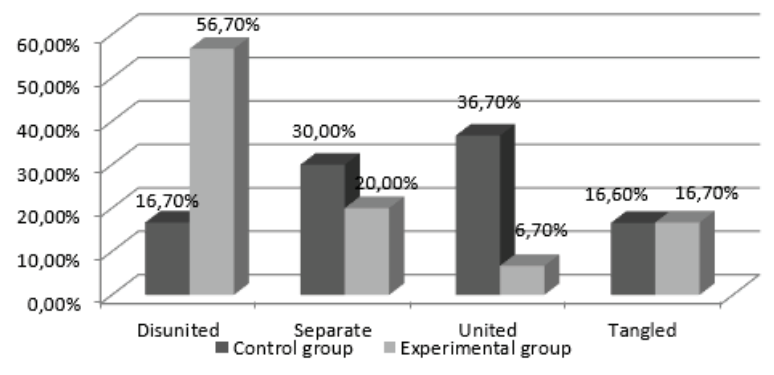

Fig. 2. Distribution according to the level of the family system cohesion, in percentage correlation.

We found that in an autistic child's family system, about half of the families have a chaotic type of adaptation, while approximately the same number of families with an ordinary child have a structured type of adaptation. We can observe this tendency of distribution of the level of adaptation in groups in the diagram in Figure 3.

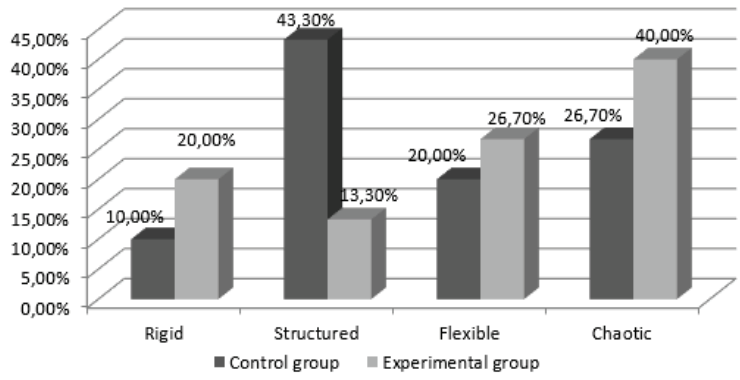

Fig. 3. Distribution of adaptation of the family system according to the questionnaire by D.Kh. Olson, J. Portner, I. Lavi, in percentage correlation 
The disengaged type of family system cohesion is characterized by alienated relations, members of such a family are often emotionally distant, have little attachment to each other. The chaotic type of adaptation of the family system has unstable or limited leadership. Decisions made in the family are often ill-conceived, roles are unclear and often shift from one family member to another. A large number of changes leads to unpredictability of what is happening in the system. Such a family system is not resilient to stress factors. A disengaged and chaotic family system is referred to a dysfunctional type of family - it is a family in which performing functions is impaired, as a result of which the goals of the family as a whole and family members in particular are not achieved in the marital, parental, material, household and other spheres of life, which interferes with the personal growth and blocks the possibility of self-actualization of family members.

It was also revealed that in the autistic children's family system, a dysfunctional type of a family statistically predominates, while semi-functional and functional types of families predominate in the family system with an ordinary child. For clarity, the diagram is shown in Figure 4.

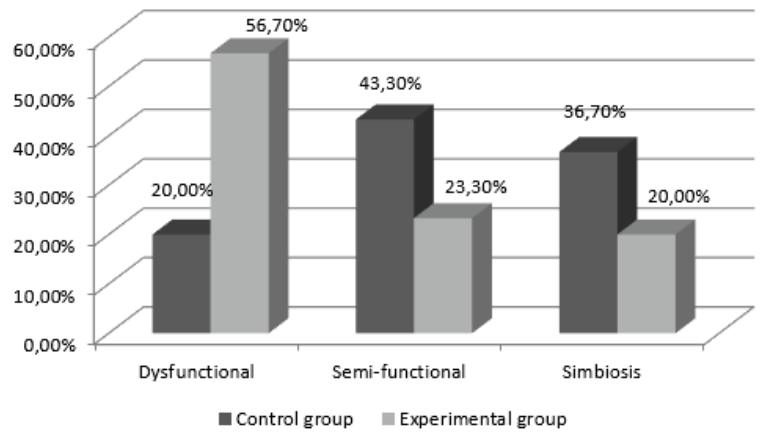

Fig. 4. Proportioning of family types by groups, in percentage correlation.

In the autistic children's family system, the number of families satisfied with the family system is almost equal to the number of families dissatisfied with the family system. At the same time, in a family system with an ordinary child, the number of people satisfied with the family system is significantly higher than the number of families dissatisfied with the family system (Fig. 5).

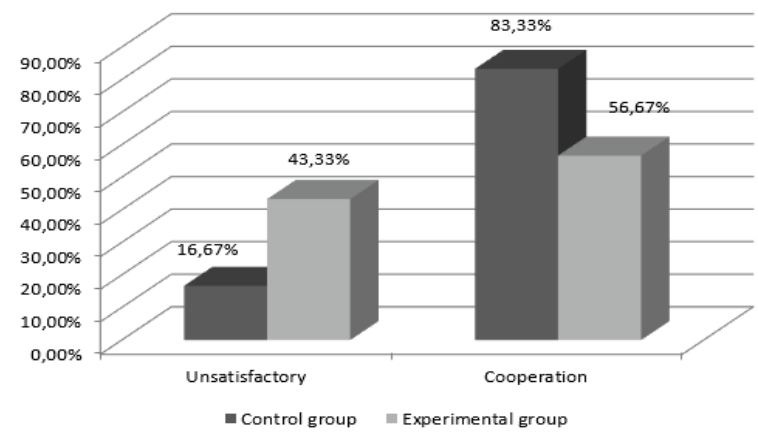

Fig. 5. Distribution of the level of satisfaction with the family system by groups, in percentage correlation.

The analysis of the distribution of the level of satisfaction with marriage in families of different types showed that the largest percentage of "bad marriages" is in families of a functional type (62.5\%); the largest percentage of "transitory marriages" is represented in 
semi-functional families (64.3\%); the highest percentage (55\%) of "good marriages" was found in dysfunctional families; the perception of one's own marriage as "absolutely successful" is more often expressed in semi-functional type families $(44.4 \%)$. The study also revealed the following tendencies:

1) in families of the dysfunctional type, the level of the control factor is statistically higher in the families in which parents are satisfied with the family system;

2) in semi-functional type of families, the level of the control factor is statistically higher in the families in which parents are not satisfied with family adaptation;

3 ) in functional type of families (and they are in the minority), the level of the control factor is the highest.

4) in the experimental group in the families of semi-functional type, along with dissatisfaction with family adaptation, we can observe an almost complete absence of symbiosis.

5 ) in the experimental group in the families of dysfunctional and semi-functional types, along with dissatisfaction with family adaptation, a decrease in the level of emotional acceptance of the child by the parents can be observed.

Thus, we can state that all the hypotheses put forward have found their confirmation. The reliability of the results obtained is confirmed through the use of diagnostic methods and techniques that are adequate to the purpose and objectives of the study, the correct use of methods of mathematical statistics.

The practical significance of this work consists in the following:

- The outcome of the study can be used for teaching disciplines revealing the nature of childhood autism.

- The results obtained can be used in practical, counseling, therapeutic work of psychologists.

- The results of the study can be used in the work of social workers.

- The results of the study can serve as the basis for the development of a program for therapeutic and preventive work with families with children having an autistic disorder.

Thus, we can conclude that the environmental specifics of the family system can have an impact on a child with autism. In this regard, it can be assumed that family therapy (working with a family symptom) could become one of the possible forms of therapy, as well as of the prevention of autistic disorder.

As a direction of further research, a deeper analysis of the family system is planned to be performed, with a possibly increased number of participants of the examination and the development of a program for therapeutic and preventive work with autistic children's families.

\section{References}

1. M.A. Bebchuk, E. Zhuykova Help to the family: psychology of decisions and changes (Independent firm "Class", Moscow, 2015)

2. B. Bettelheim, The empty fortress. Childhood Autism and Birth (Academic Project, Moscow, 2013)

3. A.Y. Varga, T.S. Drabkina, Systems family therapy. Short lecture course (Speech, St. Petersburg, 2001)

4. A.V. Chernikov, Systems family therapy: An integrative diagnostic model (Independent firm "Class", Moscow, 2001)

5. E. Eidemiller, V. Justickis, Psychology and psychotherapy of the family. (Peter, St. Petersburg, 2009) 